\title{
A Systematic Review of Healthcare Professionals' Knowledge, Self-Efficacy and Attitudes Towards Working with Autistic People
}

\author{
Kirsten Corden $^{1} \cdot$ Rebecca Brewer $^{1} \cdot$ Eilidh Cage $^{1,2}$ (D) \\ Received: 5 October 2020 / Accepted: 5 May 2021 / Published online: 14 May 2021 \\ (C) The Author(s) 2021
}

\begin{abstract}
Healthcare professionals play a vital role in identifying and supporting autistic people. This study systematically reviewed empirical research examining healthcare professionals' knowledge, self-efficacy and attitudes towards working with autistic people. Thirty-five studies were included. The included studies sampled a range of countries and professional backgrounds. A modified quality assessment tool found the quality of the included studies was moderately good. Narrative synthesis indicated that healthcare professionals report only moderate levels of autism knowledge and self-efficacy, and often lack training. Variation within and between countries and professional background was not explained by demographic factors. The reviewed evidence suggests health professionals' limited knowledge and self-efficacy in working with autistic people is a challenge to the provision of healthcare for autistic individuals.
\end{abstract}

Keywords Autism spectrum conditions $\cdot$ Healthcare professionals $\cdot$ Autism knowledge $\cdot$ Self-efficacy $\cdot$ Attitudes

Autism is a neurodevelopmental condition characterised by difficulties and differences in social communication and interaction, alongside repetitive patterns of behaviour and focused interests or activities (American Psychiatric Association, 2013). Prevalence rates have risen in recent years (Matson \& Kozlowski, 2011), with global prevalence varying from 30 in 10,000 to 116 in 10,000 of the population (Elsabbagh et al., 2012). Early identification of autism is important for facilitating any accommodations and support necessary for quality of life (Lai et al., 2014). However, recognition and diagnosis of autism predominantly rely on the knowledge of involved healthcare professionals. For example, the variabilities in symptomology, support needs and overall presentation of autism necessitate familiarity and confident interpretation of clinical characteristics according to the DSM-V (American Psychiatric Association, 2013; Baker, 2013).

This research formed part of the first author's doctorate in Clinical Psychology at Royal Holloway, University of London.

Eilidh Cage

Eilidh.cage@stir.ac.uk

1 Department of Psychology, Royal Holloway, University of London, Egham, Surrey, UK

2 Present address: Division of Psychology, Faculty of Natural Sciences, University of Stirling, Stirling, Scotland, UK
However, diagnosis is not limited to paediatric settings. Diagnosis of autism in adulthood is recognised as a clinically significant issue (National Institute for Health and Care Excellence, 2012), with a so-called lost generation of people previously excluded from a diagnosis prior to changes in the diagnostic criteria and re-conceptualisation of autism as a spectrum condition (Lai \& Baron-Cohen, 2015). Diagnosis in adulthood is crucial for increasing access to support and appropriate services but could also reduce self-criticism and foster a positive sense of identity in previously undiagnosed or misdiagnosed individuals (Hurlbutt \& Chalmers, 2002; Portway \& Johnson, 2005; Wong et al., 2015). Healthcare professionals working with autistic ${ }^{1}$ people across the lifespan and in all contexts therefore play an important role in the recognition of autism and appropriate signposting to services.

In addition to healthcare professionals' role in the diagnosis of autism, they also likely encounter autistic people in their day-to-day practice. A recent meta-analysis reported a pooled prevalence of any psychiatric condition in autistic adults as $54.8 \%$ to $60.5 \%$, dependent on the type of assessment tool used (Lugo-Marín et al., 2019). This represents a higher prevalence than in non-autistic groups (Lai et al., 2019). Limited knowledge of autism in healthcare professionals has been

\footnotetext{
${ }^{1}$ In this paper, we use identity-first terminology in line with the preferences of the autistic community (Kenny et al., 2016).
} 
identified as a key barrier to receiving appropriate psychological or therapeutic support (Jones et al., 2014). Further, higher rates of poor physical health are reported in autistic adults than non-autistic adults (46.8\% versus $23.7 \%$; Rydzewska et al., 2019). Healthcare professionals can face particular challenges in providing physical healthcare to autistic people, as core characteristics around communication and sensory sensitivities can complicate medical diagnosis and management (Kong, 2015; Schaaf et al., 2011). This illustrates the importance of healthcare professionals, in a variety of different specialities, not only possessing accurate knowledge of autism but also exhibiting self-efficacy to adapt their approach to individual patients' needs. In this context, self-efficacy refers to an individual's belief in their abilities and is based broadly on one's self-confidence.

In the general public, knowledge of autism seems to have improved over time (Dillenburger et al., 2015). A cohort study of non-autistic students demonstrated an increase in knowledge of autistic traits over a 5-year period, in addition to significantly more positive attitudes over time (White et al., 2019). However, the literature specifically on healthcare professionals, which is broadly international in origin, shows that autism knowledge is variable. For example, a study from the USA reported considerable variation in autism knowledge between different professional backgrounds, with primary care providers such as physicians and neurologists demonstrating lower levels of basic knowledge than specialists such as psychiatrists and psychologists (Heidgerken et al., 2005). Low levels of autism knowledge have also been demonstrated among nurses (Corsano et al., 2019; Igwe et al., 2011), nurse practitioners (Will et al., 2013), paediatricians (Garg et al., 2014; Kharti et al., 2011) and general practitioners (Eseigbe et al., 2015; Lian et al., 2003; Rahbar et al., 2011). Further, different cultures may have different beliefs around autism which may influence the knowledge and approach of healthcare professionals - for example, a study of Nigerian psychiatric nurses noted many believed autism was caused by curses and the devil (Bakare et al., 2009).

These studies highlight the variability in autism knowledge among healthcare professionals in different contexts, professions and cultures, and the complexity in interpreting the factors that affect this variability. It is necessary for healthcare workers to be knowledgeable of the core characteristics of autism and how they present to provide high-quality care tailored to the needs of this population (Brown \& Elder, 2014). There is, therefore, a need to examine and understand healthcare professionals' knowledge and self-efficacy of working with autistic people and the factors that affect this, such as career length, professional background and culture.

\section{The Present Review}

In summary, healthcare professionals play important and varied roles in the recognition, diagnosis and ongoing support of autistic individuals of all ages, both in relation to autism itself and to physical and mental health more broadly. To fulfil these duties, healthcare professionals require an adequate level of autism knowledge, in addition to exhibiting confidence in their skills and an open and supportive attitude to facilitate communication specific to this population's needs. The existing literature demonstrates significant variability in method, measures and results, which is difficult to interpret across disparate studies. Searches have not identified any existing review of the current evidence base. This review therefore aims to systematically examine and bring together evidence on healthcare professionals' knowledge, self-efficacy and attitudes towards working with autistic individuals.

\section{Methods}

\section{Protocol}

The methods used in this review were informed by guidance specific to undertaking systematic reviews in healthcare ('Systematic Reviews: CRD's Guidance for Undertaking Reviews in Healthcare', 2009) and followed the Preferred Reporting Items of Systematic Reviews and Meta-Analyses (PRISMA; Moher et al., 2009, 2015). A search was conducted on the International Prospective Register of Systematic Reviews (PROSPERO) in November 2019 to confirm that a similar review had not been registered previously.

\section{Eligibility}

Articles were deemed eligible for inclusion if they: (A) utilised quantitative methods; (B) utilised descriptive, cross-sectional or cohort designs; (C) sampled healthcare professionals (general practitioners, nurses, psychiatrists, doctors, psychologists or therapists); (D) utilised measures of knowledge about autism (including understanding, skills and awareness), selfefficacy (including confidence) or attitudes (including perceptions) relating to working with autistic individuals; (E) were available in English language; (F) were published in peerreviewed journals; and $(\mathrm{G})$ were published from 1994 to present, to allow for comparison of the time periods covered by the fourth and fifth editions of the DSM (American Psychiatric Association, 1994, 2013). Studies of autism in childhood and adulthood were included without differentiation.

\section{Information Sources}

One reviewer $(\mathrm{KC})$ conducted a systematic literature search in November 2019 to identify potential articles for inclusion, using the following databases: SCOPUS, PsycINFO, PubMed and Web of Science. Manual searches of the contents and reference lists of included articles were then performed. 
Where relevant full texts were unavailable, the corresponding authors were contacted to request the manuscript.

\section{Search Strategy}

The search algorithm included the following terms: health professional ("general practitioner", nurse, psychiatrist, doctor, psychologist, therapist), knowledge (skills, understanding, awareness), self-efficacy (confidence), attitude (perception, support*) and autism (autism*, Asperger*). The Boolean operator AND was used to search the three categories (health professional, knowledge and awareness and autism), and the operator OR was used to include the variants within categories. Timespan was limited to 1994 to the date of the search (November 2019). The search terms were entered into the title and abstract fields on SCOPUS, PsycINFO and PubMed, and the 'within topic' field on Web of Science.

\section{Process of Study Selection}

One reviewer $(\mathrm{KC})$ screened all titles and abstracts for inclusion against the identified eligibility criteria. All abstracts were reviewed by a second reviewer (EC) and there was excellent inter-rater reliability for inclusion ( $\alpha=99.01 \%)$. If an article was deemed relevant, the full-text manuscript was obtained and further screened.

\section{Quality Assessment Strategy}

The Newcastle-Ottawa Scale (NOS) is a widely used quality assessment tool, in part due to its brevity and ease of use, whilst also being suitable for modification (Boland et al., 2017). A degree of subjective assessment is required in using this tool, given the lack of numerical scoring system. However, a checklist system was chosen specifically due to the argument that using numerical scales for quality assessment is problematic, as they do not adequately allow for the weighting of scores for comparison (Greenland \& O'Rourke, 2001; Juni et al., 1999).

The NOS used in this review is adapted from the crosssectional version of the NOS (Modesti et al., 2016). It includes seven questions across three domains (selection, comparability and outcome), including items regarding the representativeness of the sample, the validation of measurement tools and the description and presentation of statistical tests. Items are rated as yes/'YY' (adequately addressed), partially/'P' (partially addressed) or no/'N' (not adequately addressed). The NOS also uses a 'star system', in which stars ('*') are allocated to elements suggestive of particularly high quality. The item on sample size was adapted for the present review to include a 'partially addressed' rating, to allow for distinction between studies with or without power calculations or similar sample size justification. Similarly, the item on comparability was adapted to distinguish between studies that not only identified potential confounds but additionally controlled for them. The item on non-respondents was extended to include a fourth 'non-applicable' rating, for use with studies whose convenience or opportunity sampling precluded the ability to assess responses in this way. Lastly, the item on outcome was modified to include an option for measures administered in person. The adapted NOS used in this review is included in the Supplementary Materials.

\section{Synthesis of Results}

A meta-analysis was not performed as part of this review, as the differences in methods and outcomes of the included studies varied considerably, precluding statistical synthesis. As such, a narrative synthesis of the results was considered most appropriate. The results are presented in chronological order from 1994 to the present day, to aid the interpretation of knowledge, self-efficacy and attitudes over time.

\section{Results}

Figure 1 shows the PRISMA flowchart for the systematic review. Twenty-eight out of the 2574 articles identified by the initial search and seven out of the nine articles cited within those studies were available and deemed eligible for inclusion in the review. Thirty-five studies were included overall, totalling 8398 participants from a range of healthcare backgrounds.

The results of the quality assessment of the included studies are shown in Table 1. Overall, methodological quality of the included studies was moderately good. A strength was the use of standardised measures, with comparative weaknesses in representative sampling and systematic justification of sample size.

Twelve out of 35 studies used either whole-population sampling or an element of randomisation to maximise the representativeness of the sample. The remaining 23 studies were deemed somewhat representative by the quality assessment tool since they relied on convenience sampling alone. Only five of 35 studies included either an a priori or post hoc power analysis to justify their sample size. The remaining thirty studies deemed their sample size satisfactory in relation to the estimated population size. Seventeen out of 35 studies gave a response rate, with six not mentioning non-respondents at all. Twelve studies were unable to give a response rate due to study design (i.e. convenience sampling methods). No study provided characteristics of non-respondents, which is likely to have been associated with the limitations of study design and recruitment.

Twenty-three of 35 studies used standardised measures, either validated within the study or in previous research. 
Fig. 1 PRISMA flowchart of the systematic literature review
Records identified through database search $(n=4113)$

Excluded for clearly not meeting criteria $(n=2473)$

Records after duplicates

removed $(n=2574)$
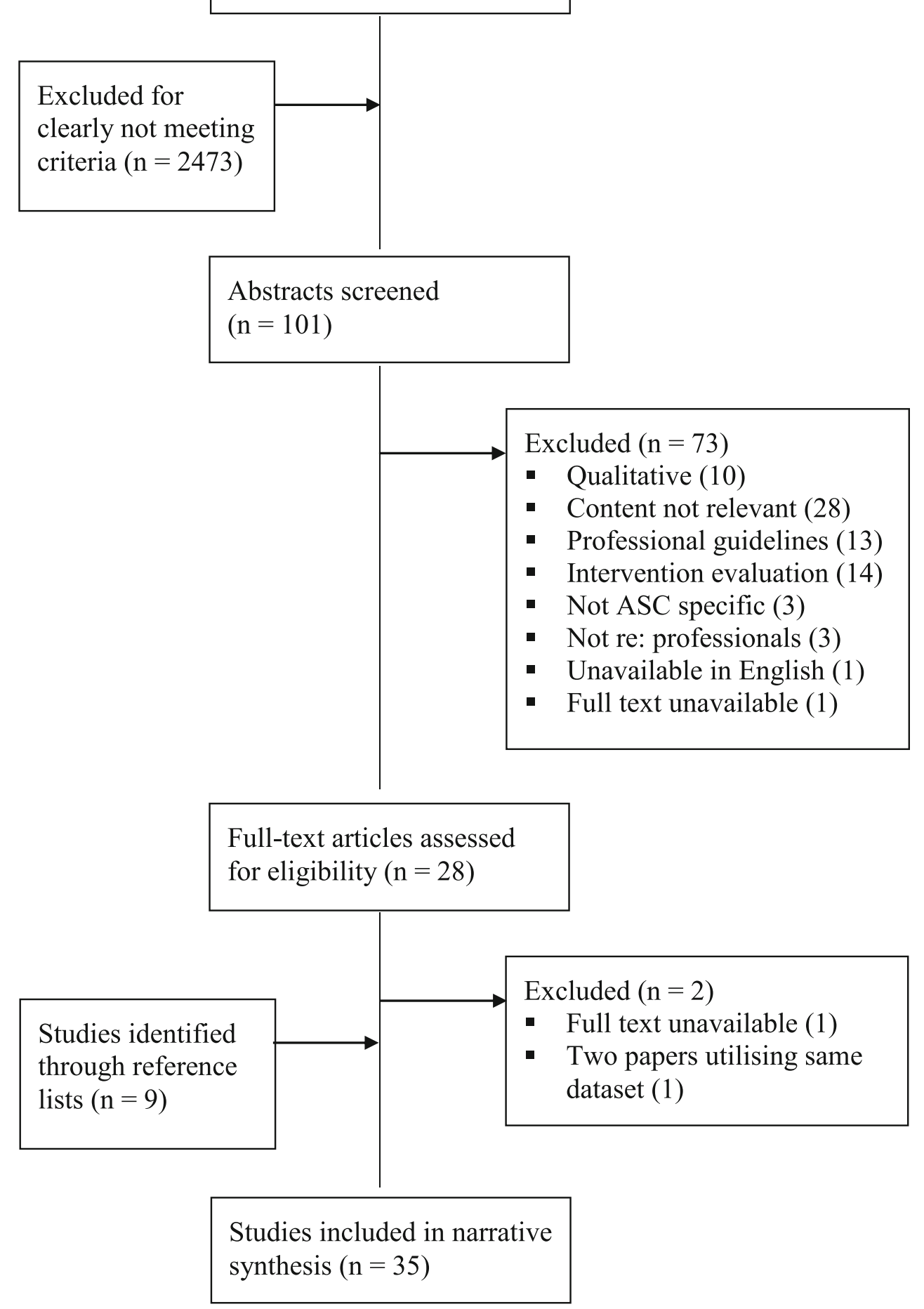
Table 1 Quality assessment of the studies included in the systematic review

\begin{tabular}{|c|c|c|c|c|c|c|c|}
\hline \multirow[b]{2}{*}{ Article } & \multicolumn{4}{|l|}{ Selection } & \multirow{2}{*}{$\begin{array}{l}\text { Comparability } \\
\text { confounding } \\
\text { factors } \\
\text { controlled }\end{array}$} & \multicolumn{2}{|l|}{ Outcome } \\
\hline & $\begin{array}{l}\text { Representative } \\
\text { of target population }\end{array}$ & Sample size & $\begin{array}{l}\text { Non- } \\
\text { respondents }\end{array}$ & Measures & & $\begin{array}{l}\text { Assessment } \\
\text { of the outcome }\end{array}$ & $\begin{array}{l}\text { Statistical } \\
\text { test }\end{array}$ \\
\hline Shah (2001) & $\mathrm{Y}^{*}$ & $\mathrm{P}$ & $\mathrm{P}$ & $\mathrm{P}^{*}$ & $\mathrm{P}$ & $\mathrm{Y}^{*}$ & $\mathrm{Y}^{*}$ \\
\hline Lian et al. (2003) & $\mathrm{P} *$ & $P$ & $P$ & $\mathrm{P}^{*}$ & $\mathrm{Y}^{*}$ & $\mathrm{Y} * *$ & $\mathrm{Y}^{*}$ \\
\hline Heidgerken et al. (2005) & $\mathrm{P} *$ & $\mathrm{P}$ & $\mathrm{N}$ & $\mathrm{Y}^{* *}$ & $\mathrm{P}$ & $\mathrm{Y}^{*}$ & $\mathrm{Y}^{*}$ \\
\hline Bakare et al. (2008) & $\mathrm{P} *$ & $\mathrm{P}$ & N/A & $\mathrm{Y}^{* *}$ & $\mathrm{P}$ & $\mathrm{Y}^{* *}$ & $\mathrm{Y}^{*}$ \\
\hline Bakare et al. (2009) & $\mathrm{Y}^{*}$ & $\mathrm{P}$ & N/A & $\mathrm{Y}^{* *}$ & $\mathrm{Y}^{*}$ & $\mathrm{Y}^{* *}$ & $\mathrm{Y}^{*}$ \\
\hline Strunk (2009) & $\mathrm{P} *$ & $\mathrm{P}$ & $\mathrm{P}$ & $\mathrm{Y}^{* *}$ & $\mathrm{P}$ & $\mathrm{Y}^{*}$ & $\mathrm{~N} / \mathrm{A}$ \\
\hline Self et al. (2010) & $\mathrm{Y}^{*}$ & $\mathrm{P}$ & $\mathrm{P}$ & $\mathrm{Y}^{* *}$ & $\mathrm{P}$ & $\mathrm{Y}^{*}$ & $\mathrm{Y}^{*}$ \\
\hline Igwe et al. (2010) & $\mathrm{Y}^{*}$ & $\mathrm{P}$ & N/A & $\mathrm{Y}^{* *}$ & $\mathrm{Y}^{*}$ & $\mathrm{Y}^{*}$ & $\mathrm{P}$ \\
\hline Imran et al. (2011) & $\mathrm{P}^{*}$ & $\mathrm{P}$ & $\mathrm{P}$ & $\mathrm{P} * / \mathrm{Y} * * a$ & $\mathrm{P}$ & $\mathrm{Y}^{*}$ & $\mathrm{P}$ \\
\hline Igwe et al. (2011) & $\mathrm{Y}^{*}$ & $\mathrm{P}$ & N/A & $\mathrm{Y}^{* *}$ & $\mathrm{Y}^{*}$ & $\mathrm{Y}^{*}$ & $\mathrm{Y}^{*}$ \\
\hline Rahbar et al. (2011) & $\mathrm{Y}^{*}$ & $\mathrm{Y}^{*}$ & $\mathrm{~N}$ & $\mathrm{P}^{*}$ & $\mathrm{P}$ & $\mathrm{Y}^{* *}$ & $\mathrm{Y}^{*}$ \\
\hline Kharti et al. (2011) & $\mathrm{Y}^{*}$ & $\mathrm{P}$ & $\mathrm{P}$ & $\mathrm{N}$ & $\mathrm{N}$ & $\mathrm{Y}^{*}$ & $\mathrm{Y}^{*}$ \\
\hline Muhammad et al. (2013) & $\mathrm{Y}^{*}$ & $P$ & N/A & $\mathrm{Y}^{* *}$ & $\mathrm{~N}$ & $\mathrm{Y} * *$ & $\mathrm{Y}^{*}$ \\
\hline Will et al. (2013) & $\mathrm{P} *$ & $\mathrm{Y}^{*}$ & N/A & $\mathrm{P} * / \mathrm{Y} * *{ }_{\mathrm{a}}$ & $\mathrm{P}$ & $\mathrm{Y}^{*}$ & $\mathrm{P}$ \\
\hline Garg et al. (2014) & $\mathrm{Y}^{*}$ & $\mathrm{Y}^{*}$ & $\mathrm{P}$ & $\mathrm{Y} * *$ & $\mathrm{P}$ & $\mathrm{Y}^{*}$ & $\mathrm{Y}^{*}$ \\
\hline James et al. (2014) & $\mathrm{P} *$ & $\mathrm{P}$ & N/A & $\mathrm{Y}^{* *}$ & $\mathrm{P}$ & $\mathrm{Y}^{*}$ & N/A \\
\hline Shaukat et al. (2014) & $\mathrm{Y}^{*}$ & $\mathrm{Y}^{*}$ & $\mathrm{P}$ & $\mathrm{Y}^{* *}$ & $\mathrm{Y}^{*}$ & $\mathrm{Y} * *$ & $\mathrm{P}$ \\
\hline Zerbo et al. (2015) & $\mathrm{P} *$ & $\mathrm{P}$ & $\mathrm{P}$ & $\mathrm{P}^{*}$ & $\mathrm{P}$ & $\mathrm{Y}^{*}$ & $\mathrm{P}$ \\
\hline Eseigbe et al. (2015) & $\mathrm{P} *$ & $\mathrm{P}$ & $P$ & $\mathrm{Y}^{* *}$ & $\mathrm{~N}$ & $\mathrm{Y}^{*}$ & $\mathrm{P}$ \\
\hline Bakare et al. (2015) & $\mathrm{Y}^{*}$ & $\mathrm{P}$ & N/A & $\mathrm{Y}^{* *}$ & $\mathrm{Y}^{*}$ & $\mathrm{Y}^{* *}$ & $\mathrm{P}$ \\
\hline Murphy and McMorrow (2015) & $\mathrm{P} *$ & $P$ & $\mathrm{P}$ & $\mathrm{P}^{*}$ & $\mathrm{P}$ & $\mathrm{Y}^{*}$ & N/A \\
\hline Lüleci et al. (2016) & $\mathrm{P} *$ & $\mathrm{P}$ & $\mathrm{P}$ & $\mathrm{Y}^{* *}$ & $\mathrm{P}$ & $\mathrm{Y}^{* *}$ & $\mathrm{Y}^{*}$ \\
\hline Clark et al. (2016) & $\mathrm{P} *$ & $\mathrm{P}$ & $\mathrm{N}$ & $\mathrm{P}^{*}$ & $\mathrm{~N}$ & $\mathrm{Y}^{*}$ & N/A \\
\hline Williams and Haranin (2016) & $\mathrm{P} *$ & $\mathrm{P}$ & $\mathrm{P}$ & $\mathrm{P} *$ & $\mathrm{P}$ & $\mathrm{Y}^{*}$ & $\mathrm{Y}^{*}$ \\
\hline Gardner et al. (2016) & $\mathrm{Y}^{*}$ & $\mathrm{P}$ & $\mathrm{N}$ & $\mathrm{Y}^{* *}$ & $\mathrm{P}$ & $\mathrm{Y}^{*}$ & $\mathrm{P}$ \\
\hline Unigwe et al. (2017) & $\mathrm{P}^{*}$ & $\mathrm{P}$ & N/A & $\mathrm{Y}^{* *}$ & $\mathrm{~N}$ & $\mathrm{Y}^{*}$ & $\mathrm{Y}^{*}$ \\
\hline Johnsson et al. (2017) & $\mathrm{P} *$ & $\mathrm{P}$ & N/A & $\mathrm{N}$ & $\mathrm{N}$ & $\mathrm{Y}^{*}$ & N/A \\
\hline Cooper et al. (2018) & $\mathrm{P} *$ & $\mathrm{P}$ & $\mathrm{P}$ & $\mathrm{Y}^{* *}$ & $\mathrm{P}$ & $\mathrm{Y}^{*}$ & $\mathrm{Y}^{*}$ \\
\hline Sampson and Sandra (2018) & $\mathrm{P} *$ & $\mathrm{P}$ & $P$ & $\mathrm{Y}^{* *}$ & $\mathrm{Y}^{*}$ & $\mathrm{Y}^{* *}$ & $\mathrm{P}$ \\
\hline Atun-Einy and Ben-Sasson (2018) & $\mathrm{P} *$ & $P$ & N/A & $\mathrm{Y}^{* *}$ & $\mathrm{P}$ & $\mathrm{Y}^{*}$ & $\mathrm{Y}^{*}$ \\
\hline Ellias and Shah (2019) & $\mathrm{P} *$ & $\mathrm{Y}^{*}$ & $\mathrm{~N}$ & $\mathrm{Y}^{* *}$ & $\mathrm{P}$ & $\mathrm{Y}^{*}$ & $\mathrm{Y}^{*}$ \\
\hline Hayat et al. (2019) & $\mathrm{P}^{*}$ & $\mathrm{P}$ & $\mathrm{N}$ & $\mathrm{Y}^{* *}$ & $\mathrm{Y}^{*}$ & $\mathrm{Y}^{* *}$ & $\mathrm{P}$ \\
\hline Austriaco et al. (2019) & $\mathrm{P}^{*}$ & $\mathrm{P}$ & $\mathrm{P}$ & $\mathrm{N}$ & $\mathrm{P}$ & $\mathrm{Y}^{*}$ & $\mathrm{Y}^{*}$ \\
\hline Corsano et al. (2019) & $\mathrm{P} *$ & $\mathrm{P}$ & $\mathrm{P}$ & $\mathrm{Y}^{* *}$ & $\mathrm{Y}^{*}$ & $\mathrm{Y}^{* *}$ & $\mathrm{Y}^{*}$ \\
\hline Crane et al. (2019) & $\mathrm{P}^{*}$ & $\mathrm{P}$ & N/A & $\mathrm{Y}^{* *}$ & $\mathrm{P}$ & $\mathrm{Y}^{*}$ & $\mathrm{Y}^{*}$ \\
\hline
\end{tabular}

$Y$ yes (item adequately addressed); $P$ partially (item partially addressed); $N$ no (item not adequately addressed). Stars $(*)$ are allocated to elements suggestive of particularly high quality. ${ }_{a}$ Studies rated as $\mathrm{P}^{*} / \mathrm{Y}^{* *}$ for measures include a mixture of validated and non-validated measures

Seven studies used non-validated measures, designed by the authors for their specific study, with the measure included in appendices or adequately described for replication. Two studies used a mixture of validated and non-validated measures. 
The remaining three studies did not provide or adequately describe the measurement tools used.

Nine out of 35 studies identified and controlled for potential confounds within the study design or analysis, such as sampling method, reporting bias and validity or reliability of measures used. Controls included total population sampling to maximise generalisability of results and research staff supervising the completion of measures to ensure respondents did not have access to answers to knowledge questions. A further twenty studies identified potential confounds but did not control for these. Six studies did not mention confounds in either the method or discussion sections of the articles.

In view of assessment methodology, 11 out of 35 studies were conducted via interview, or with the self-report measures administered in person under controlled conditions. The other 24 studies were completed with self-report measures distributed via post, email or online survey software.

Statistical analyses were appropriate and clearly described in 20 of 35 studies, whilst incomplete reporting of test statistics and results lowered the quality for a further ten studies. The remaining five studies did not conduct any inferential statistical analyses and reported frequency data alone.

\section{Study Characteristics}

Characteristics and results of the final 35 studies included in the review are presented in the Supplementary Materials. Studies were published between 2001 and 2019, with 11 from 2001 to 2012 and 24 from 2013 to 2019 , relating to the active periods of the DSM-IV and DSM-V (APA, 1994, 2013), respectively. The studies reflect research from a range of countries, including the USA (9), the UK (6), Nigeria (6), Pakistan (3), Australia (2), Ghana, India, Iraq, Israel, Italy, Nepal, Saudi Arabia, Singapore and Turkey. All studies used a cross-sectional design, with the exception of Bakare et al. (2008), who used a within-participants repeated measures design for scale validation over a period of 2 weeks. Data were collected through self-report measures (34) or structured interview (1). Sample size ranged from 19 to 1396 (median sample size $=175$ ). Of the studies that categorised participants by professional background, samples included medical students (1656), occupational therapists (OTs; 1536), nurses (1098), general practitioners (GPs; 891), unspecified doctors (421), psychiatrists (172), physiotherapists (151), psychological therapists (143), speech and language therapists (SLTs; 129), nursing students (100) and psychology students (100). Psychology students were included in the sample as in one study they were compared to other healthcare students, and despite not working as health professionals in the UK, students often go into graduate jobs with clinical responsibility in other countries, such as Nigeria. Recruitment sources varied, with most studies using local hospitals or healthcare facilities (11) or via email and online methods (9). Participants were also recruited through meetings or conferences (6), local universities (5), professional bodies (1) and a mail distribution list (1). It was unclear how the remaining two studies recruited their samples.

Fourteen of the studies framed their questions and findings in terms of autism in children, with the remaining 21 studies reflecting generic knowledge about autism at any age. Twenty-seven studies addressed healthcare professionals' knowledge of autism, with six exploring self-efficacy or confidence, five investigating training backgrounds and two surveying attitudes towards autism. The most frequently used measure was the Knowledge About Childhood Autism Among Health Workers Scale (KCAHW; Bakare et al., 2008). Four studies used the Autism Survey (Stone, 1987) and two used an updated version re-named the Knowledge of Autism Scale (Unigwe et al., 2017). Measures of self-efficacy, training experiences and attitudes were developed by the authors for each individual study.

\section{Study Findings}

Knowledge Most studies focused on knowledge of the core characteristics of autism. Studies using the KCAHW (Bakare et al., 2008) reported mean knowledge scores ranging from 9.01 to 13.5 out of a maximum of 19 , translating to between 47.37 and $71.05 \%$ correct answers. These results represent large variation in knowledge of autism across the included literature, with even the higher scores suggesting room for improvement in knowledge.

In the remaining studies, $62 \%$ of GPs in Australia achieved $75 \%$ correct answers or higher on a novel measure of autism knowledge (Garg et al., 2014). In the USA, $85 \%$ of prequalified medical students rated their knowledge as 'somewhat informed' or below (Austriaco et al., 2019), a figure mirrored by qualified clinicians in the USA, of whom $77 \%$ rated their knowledge as 'poor' to 'fair' (Zerbo et al., 2015). Awareness rates also showed large variations between studies, with $44.6 \%$ of general practitioners in Pakistan reporting that they had heard of autism (Rahbar et al., 2011), compared to 95\% of doctors in Iraq (Muhammad et al., 2013). Recognition of diagnostic criteria also varied, ranging from 35.2 of doctors in Nepal demonstrating 'adequate' knowledge of diagnosis (Kharti et al., 2011) to $73.67 \%$ of paediatric staff in Israel from different professional backgrounds (Atun-Einy \& BenSasson, 2018).

Four out of five studies that explored the relationship between knowledge and gender reported no significant relationship (Bakare et al., 2015; Lian et al., 2003; Rahbar et al., 2011; Shaukat et al., 2014), and one study showed significantly higher levels of autism awareness in females than males (Lüleci et al., 2016). Conflicting results were found for the relationship between knowledge and age in the six studies that reported these statistics. Whilst one study found that 
knowledge scores increased with age (Corsano et al., 2019), two studies found that knowledge scores were higher among younger staff (Garg et al., 2014; Rahbar et al., 2011) and the three remaining studies found no significant difference by age (Igwe et al., 2011; Lian et al., 2003; Unigwe et al., 2017). Two studies reported a positive relationship between personal experience of autism (i.e. having autistic friends or family) and knowledge scores (Crane et al., 2019; Unigwe et al., 2017).

Three out of four studies found a positive relationship between completion of autism-specific training or education programmes and knowledge scores (Austriaco et al., 2019; Crane et al., 2019; Williams \& Haranin, 2016), and one study found no relationship (Unigwe et al., 2017). Seven studies reported that knowledge of autism improved with the length of experience of working with autistic clients (Austriaco et al., 2019; Bakare et al., 2009, 2015; Corsano et al., 2019; Hayat et al., 2019; Kharti et al., 2011; Sampson \& Sandra, 2018), whilst two studies reported that those with less experience or newly qualified had better knowledge (Garg et al., 2014; Rahbar et al., 2011). Furthermore, three studies reported no significant relationship between length of experience working with autistic individuals and knowledge scores (Cooper et al., 2018; Igwe et al., 2011; Unigwe et al., 2017).

Finally, out of the studies that reported knowledge scores by profession, four studies reported low autism knowledge in general practitioners, physicians and adult health providers (Eseigbe et al., 2015; Gardner et al., 2016; Hayat et al., 2019; Imran et al., 2011). Paediatric or psychiatric specialties were associated with greater knowledge of autism in five studies (Bakare et al., 2015; Eseigbe et al., 2015; Gardner et al., 2016; Hayat et al., 2019; Sampson \& Sandra, 2018). Two studies reported significant differences between professions or specialties, but specific statistics were not reported (Igwe et al., 2011; Zerbo et al., 2015).

Self-Efficacy Three studies explored professionals' selfefficacy around working with autistic individuals in relation to other factors. Higher self-efficacy related to greater levels of knowledge about autism (Atun-Einy \& Ben-Sasson, 2018; Crane et al., 2019), a higher proportion of autistic clients on caseload (Crane et al., 2019; Williams \& Haranin, 2016), more experience of working with autistic clients (Atun-Einy \& Ben-Sasson, 2018) and receiving autism-specific training either during or after qualification (Crane et al., 2019; Williams \& Haranin, 2016).

Three studies reported on professionals' confidence about working with autistic people. Different measures were used in each study, but converting mean scores into percentages illustrated comparable results, with moderate levels of confidence reported throughout $(62.25 \%$ (Atun-Einy \& Ben-Sasson, 2018); 66.5\% (Johnsson et al., 2017); 59.2\% (Cooper et al., 2018)). Johnsson et al. (2017) identified low levels of confidence in managing 'challenging behaviours' associated with autism and addressing families' questions and concerns. Furthermore, Will et al., (2013) found that self-rated competency scores for working with autistic people was significantly lower than for working with non-autistic individuals with other chronic or complex conditions.

Attitudes Two studies reported on professionals' comfort in working with autistic people. Out of ten, medical students rated their comfort in treating autistic people as 4.61 on average, compared to 6.63 in a more qualified cohort of medical trainees (Austriaco et al., 2019). Gardner et al. (2016) reported 'over half' of their participants described feeling 'uncomfortable' or 'slightly uncomfortable' in treating autistic people, with $79 \%$ describing treating autistic people as 'difficult' or 'very difficult'. Two further studies explored professionals' beliefs about autism. Bakare et al. (2009) found that $41 \%$ of healthcare workers in Nigeria attributed autism to preternatural causes, including lineage curses, enemies and the devil. Further, $32.1 \%$ of the sample believed that autism was preventable through avoiding maternal infections, birth injuries or sin and pleasing ancestral spirits. The study found these beliefs were significantly related to lower levels of healthcare experience. Heidgerken et al. (2005) reported that primary care providers in the US (i.e. family practitioners, paediatric and neurology specialists) were more likely to describe autistic children as 'negativistic' and 'non-compliant' and to attribute behaviours to 'cold, rejecting parents' than autism practitioners.

Results by Time Period Due to the variation in method and measures, it is not possible to statistically compare all the results across the two periods in question. However, a comparison can be made of autism knowledge from the eleven studies utilising the KCAHW (Bakare et al., 2008). The three studies from the period covered by the DSM-IV (1994-2012; APA, 1994) had a mean of 11.86 (out of 19), with a range of 10.67 to 12.56 . The eight studies from the period covered by the DSM-V (2013-present; APA, 2013) had a mean of 12.04 (out of 19), with a range of 9.8 to 13.5 .

\section{Discussion}

This study systematically reviewed existing quantitative research examining healthcare professionals' knowledge of autism, in addition to their self-efficacy and attitudes towards working with autistic people. Thirty-five met our inclusion criteria. The studies covered a range of professional backgrounds, populations, measures used and countries of origin. Overall, the collated results indicated low to moderate levels of knowledge and perceived self-efficacy about autism among health workers across a range of backgrounds. However, there was disparity of results between studies, both domestic and 
international in context. For example, this variation is illustrated by comparing four UK studies, in which responses ranged from 88.1 to $91.2 \%$ correct answers for knowledge in general practitioners, community clinical staff and psychiatrists (Clark et al., 2016; Crane et al., 2019; Unigwe et al., 2017), whereas one UK study found that only $28 \%$ of patient-facing clinical staff (including nurses, psychiatrists and psychologists) reported adequate autism knowledge (Murphy \& McMorrow, 2015). Taken together, these results indicate that autism knowledge is highly variable across samples and individuals - and even in samples with higher scores, there is still room for improvement.

Most studies attempted to examine associations between knowledge, self-efficacy and attitudes towards autism in relation to demographic factors, such as professional background, age and gender of the participants, in addition to length of professional experience and experiences of autism-specific training. However, the studies gave a conflicting narrative, perhaps complicated by methodological differences between the studies and the range of measures used. Overall, the results tended to suggest that there was no significant relationship between autism knowledge and gender of the respondents. Seven of nine studies reported greater knowledge of autism with increased experience, which potentially reflects individuals' knowledge improving over time. The studies that compared results between professional backgrounds indicated variations by specialty, with general practitioners and adult health providers tending to report lower levels of knowledge and self-efficacy, and paediatric and psychiatric specialties reporting higher scores overall. Potentially, this could be indicative of the breadth of content in general medical training compared to the relative specialisation for paediatric and psychiatric medicine. Likewise, a positive relationship was observed between autism knowledge and perceived self-efficacy, which could be understood in terms of increased knowledge potentially leading to greater confidence in skills, which then increases an individual's belief in their capacity to perform specific behaviours in their clinical practice.

The studies included a range of countries with a variety of belief systems. Perhaps the most striking example of cultural influence was described in Bakare et al.'s (2009) study of community psychiatric nurses in Nigeria. In this study, over $40 \%$ of respondents attributed the aetiology of autism to preternatural causes, including lineage curses, enemies and the devil. Further, over $30 \%$ of the sample believed autism was preventable, with potential mechanisms including avoiding sin or pleasing ancestral spirits. Although endorsement of these beliefs was not significantly related to sociodemographic variables, they were increasingly reported by nurses with less than six years' experience. This finding suggests that learning and experience post-qualification might influence healthcare worker's belief systems. Interestingly, primary care providers in the USA, including family and paediatric specialists, endorsed beliefs that autistic children are 'negativistic' and 'noncompliant', in addition to attributing withdrawal behaviours to "cold, rejecting parents" (Heidgerken et al., 2005). Similar beliefs were also reported by paediatricians in India, with over $20 \%$ believing autism could be caused by child neglect (Kharti et al., 2011). Not only are these beliefs and attitudes highly incongruent with current research and medical guidance (e.g. APA, 2013; NICE, 2016, 2017), they are especially concerning when reported among professional specialties, who may have regular contact with autistic people and their families.

Lastly, this review was interested in whether there was variation in knowledge, self-efficacy and attitudes towards autism over time. This approach was to acknowledge the increased prevalence of autism diagnoses in recent decades (Lyall et al., 2017), in addition to considering the changes in diagnostic criteria in the same time period. The impact of these factors on healthcare professionals' knowledge and training is relatively unknown. A comparison of levels of knowledge from studies using the same standardised measure suggest a minor improvement in scores from the period of 1994 to 2013 (mean $=11.86$ ) to the period of 2013 to the present day (mean $=12.37$ ). However, these results are only based on 11 out of the 35 studies included in the review, so it is unclear to what extent they are generalisable. No studies post-2013 explored professionals' beliefs about autism. Similarly, there were no studies in the 1994 to 2013 time period which assessed selfefficacy or attitudes towards working with autistic individuals. However, it was noted in post-2013 studies that participants described feeling discomfort or lack of confidence in treating autistic people (Austriaco et al., 2019; Cooper et al., 2018; Gardner et al., 2016; Johnsson et al., 2017; Murphy \& McMorrow, 2015; Williams \& Haranin, 2016).

\section{Strengths and Limitations of the Data}

The overall pattern of results was somewhat consistent across all studies included in the review, despite the range of professions, measures and settings sampled. It is noted, however, that the international studies highlighted clear differences in terms of respondents' core beliefs about autism. It could be argued, therefore, that cultural differences might explain the low levels of autism knowledge more in some countries relative to others, but the overall trend of relatively low to moderate knowledge on autism remained similar, suggesting there is room for improvement in knowledge across all cultures and professions. One of the strengths of the data, therefore, is that it is possible to draw some conclusions about healthcare professionals' general levels of knowledge, self-efficacy and attitudes towards autism and providing care to autistic people. However, it should be noted that most studies focused on general knowledge of core autism characteristics, rather than testing knowledge of co-occurring conditions or knowledge 
within a healthcare context. For example, the most used knowledge measure (KCAHW, Bakare et al., 2008) does not examine knowledge of providing appropriate and accessible care to autistic people. Qualitative research indicates that healthcare providers acknowledge the need to adapt the sensory environment, develop effective communication and the need for more training and partnership (Zwaigenbaum et al., 2016). Developing appropriate healthcare should be done in conjunction with autistic people themselves, with the Academic Autism Spectrum Partnership in Research and Education (AASPIRE) providing a leading example of this approach (e.g. Nicolaidis et al., 2016). Future studies should consider examining healthcare professional's knowledge of effectively supporting autistic people in healthcare settings and the treatment of co-occurring conditions.

The quality of the papers was moderately good overall, demonstrating strengths in the measures used and potential confounds identified and controlled for where possible. Most studies utilised standardised measures in their assessments, with several reporting the validation process. Considering the reliability and validity of these measures was vital for assessing whether the results were meaningful and adequately represented the identified concepts. This approach allowed for relative confidence in the collation and comparison of results between studies. Likewise, several authors were transparent about the potential limitations of their self-report measures and identified ways to maximise returns and encourage responses reflective of participants' actual knowledge, such as completion under supervision. There were weaknesses in sample size justifications, however, with few studies considering or calculating adequate power for their designs. This limitation does suggest that caution should be used when interpreting the results of studies with small sample sizes, or when multiple professional groups were sampled for comparison. Similarly, several papers did not report their analyses or results in full, which led to some difficulties in interpretation. In the future, we recommend that researchers ensure rigor in the application and reporting of statistical concepts in all stages of their research, to strengthen the evidence base and ensure accurate interpretation.

\section{Strengths and Limitations of the Review}

The review process demonstrates several components necessary for a good-quality, comprehensive systematic review, including a clear and replicable search strategy and quality assessment process (Boland et al., 2017). The high level of agreement between two independent reviewers $(\alpha=99.01)$ illustrated that inclusion and exclusion criteria were implemented correctly and consistently. In terms of the articles included in the review, twenty-eight eligible papers were identified from the initial database search, with seven eligible papers identified through subsequent manual search of references lists. All seven papers included key words used in the search strategy in their titles and abstracts, including "knowledge", "autism" and "health". This indicates that the search terms did adequately capture the relevant concepts and therefore does not explain why the seven papers were not identified. However, on inspection, at least three of the seven articles identified in the manual search were published in international journals, which may not have been included in the databases used. It may be possible that the remaining four papers were not identified in the database search for similar reasons unrelated to the search strategy. However, this limitation is not deemed problematic, as manual searches are intended and recommended to increase sensitivity of the search and minimise retrieval bias (Chapman et al., 2010; Greenhalgh \& Peacock, 2005).

It was not possible to obtain two papers, one identified during the initial database search (Mohammed \& Majeed, 2019) and one during the manual search of reference lists (Hartley-McAndrew et al., 2016). Multiple methods were utilised to access the full-text articles, including through two University Libraries, Google Scholar and Research Gate and contacting the corresponding authors. The availability of these papers could potentially have had an impact on the pattern of results, if eligible for inclusion. For example, if these papers had presented high levels of knowledge, confidence and positive attitudes from healthcare professionals, this would have contributed to an increasingly mixed pattern of findings. However, given the relatively small number of unavailable papers (2) to available papers (35), the potential impact on the synthesis of results and overall narrative is deemed small.

In considering how the search strategy may have influenced retrieval bias, a potential limitation was identified in the exclusion of unpublished studies and theses. Nonsignificant results in psychological and psychiatric research are less likely to be published, which may skew the published evidence base towards a particular trend of results (Joober et al., 2012). It is noted, however, that the studies in this review contained both significant and non-significant results. Similarly, through the exclusion of non-English language papers, there could be cultural bias in the collated results. However, this review did identify papers from a range of international origins, encapsulating variations in culture and attitude. Further, the systematic review excluded qualitative studies, which may mean that valuable insights are not captured in this review. Intervention studies which aim to improve knowledge, attitudes or self-efficacy in healthcare professionals were also not included, but further systematic reviews of such interventions would be highly beneficial.

Another limitation is that the approach in aggregating data across professions may miss some of the nuances of knowledge, self-efficacy and attitudes within particular disciplines. Different healthcare professionals will have different specialties which may give them stronger knowledge in some 
areas - for example, psychiatrists may have better knowledge of co-occurring mental health conditions. Some studies included in this review did note differences between professions, as discussed in our analysis, which does suggest that there is variation between disciplines. Notably, general practitioners tended to have poorer knowledge, which could be problematic since autistic individuals may be more likely to have contact with general, non-specialist providers than specialist services (Mason et al., 2019). Further, this review may miss analysis of healthcare professional's knowledge of autism in individuals who are female, late-diagnosed, nonCaucasian or at the intersection of different identities. It is important that we understand whether healthcare professionals from different backgrounds have up-to-date knowledge and confidence in supporting all autistic people.

Finally, the quality assessment tool used in this study was specifically adapted from the cross-sectional version of the NOS (Modesti et al., 2016). This approach poses potential strengths and challenges in evaluating the review process. The modified version of the tool is unstandardised, and therefore, its reliability and validity is unknown. This is tempered, however, by the minor nature of the modifications (e.g. including a grade of 'partially addressed') and the tailoring of the tool to ensure a more meaningful quality assessment of the data (Boland et al., 2017).

\section{Implications}

It is likely that healthcare professionals' knowledge of autism and confidence in their skills to work with autistic people impacts on their provision of care. Depending on their roles, this care may include decisions to refer autistic people for diagnosis and ongoing support, both relating to autism itself and co-occurring conditions (Hedley et al., 2016), which potentially puts autistic people at risk of not receiving adequate healthcare (C. Nicolaidis et al., 2012; Saqr et al., 2017). Indeed, autistic children are at significantly higher risk for having unmet health and therapy care needs than children with comparable emotional, developmental or behavioural needs, with providers' lack of skills cited as a barrier in obtaining specialist care (Chiri \& Warfield, 2012). This finding is of concern, as high rates of physical illness and mental health difficulties are consistently reported in autistic individuals (Lever \& Geurts, 2016; Rydzewska et al., 2019). Studies have reported, for example, elevated levels of epilepsy, sleep problems, allergies, gastrointestinal conditions and sensory impairments such as vision or hearing loss in autistic samples (Amiet et al., 2008; Croen et al., 2015; Gotham et al., 2015; Jones et al., 2016; Rydzewska et al., 2019), as well as higher prevalence of neurodevelopmental and mental health conditions such as depression, anxiety, eating disorders and ADHD (e.g. Hofvander et al., 2009). Overall, autistic people across the lifespan report significantly lower physical and mental health-related quality of life than their non-autistic counterparts (Khanna et al., 2014; Kuhlthau et al., 2013). Areas of research that have been suggested for prioritisation include understanding the needs of autistic adults around health and aging, psychological and pharmacological therapies, plus lifespan appropriate treatment and support (Murphy et al., 2016). It is vital that healthcare professionals, within all disciplines, have a good understanding of these co-occurring conditions for autistic people. A greater evidence base for the assessment and treatment of co-occurring conditions amongst autistic people would be beneficial and ultimately potentially enhance confidence and self-efficacy in healthcare providers.

Encouragingly, the Westminster Commission on Autism (2016) identified workforce training and access to diagnostic and support services as a key priority in the UK. However, the variation in results reported in the UK and international literature identified in this systematic review make it unclear whether adequate progress is being made. As such, there are several recommendations that arise from this review. First, efforts to enhance knowledge and perceived self-efficacy are critical for improving autistic people's access to services. Gaps in autism training during professional education, for all healthcare disciplines, should be addressed, for all clinicians to develop a basic foundation of knowledge, confidence and self-efficacy in working with autistic individuals. This is important both in terms of diagnosis and support that relate directly to autism itself, as well as providing support for other co-occurring conditions, and understanding how autism may interact with additional diagnoses, especially mental health conditions.

Trials of interventions to boost knowledge, self-efficacy and attitudes towards working with autistic people should be conducted and evaluated in different healthcare settings, which may prove beneficial to those already qualified. For example, a pilot online interprofessional training programme for allied health professionals resulted in a significant increase in scores of basic knowledge of autism and favourable attitudes to autistic people from pre-test to post-test (Beverly $\&$ Wooster, 2018). Similar positive results have been found in a randomised controlled trial of an educational curriculum delivered to family nurse practitioner students (Iannuzzi et al., 2019) and in a brief training programme for medical technicians, paramedics and nurses around providing emergency care for autistic people (McGonigle et al., 2014). More empirical evidence is needed, however, on how professionals within all disciplines can improve their knowledge and selfefficacy. Until such programmes of improvements are implemented, tested for feasibility and consistently maintained across different professional backgrounds, it will continue to be challenging for health services to champion the necessary availability and accessibility to health preventions, management and support that are tailored to the needs of this population. 


\section{Conclusion}

This paper systematically reviewed the quantitative literature exploring healthcare professionals' knowledge, self-efficacy and attitudes to autism and providing care for autistic people. The resulting evidence suggests that many of these professionals report only moderate levels of knowledge and selfefficacy in their practice, and often lack autism-specific training in their professional education. Although there is some variation in results between countries and the periods of diagnostic change from the early 2000s to the present day, limited knowledge and a lack of comfort and self-efficacy in working with autistic people may continue to be a significant barrier for autistic people accessing healthcare.

Supplementary Information The online version contains supplementary material available at https://doi.org/10.1007/s40489-021-00263-w.

Authors' Contributions Kirsten Corden: Conceptualisation, formal analysis, investigation, data curation, writing original draft. Rebecca Brewer: Conceptualisation, writing - review and editing - supervision. Eilidh Cage: Conceptualisation, validation, writing - review and editingsupervision.

\section{Declarations}

Conflict of Interest The authors declare that they have no conflict of interests.

Open Access This article is licensed under a Creative Commons Attribution 4.0 International License, which permits use, sharing, adaptation, distribution and reproduction in any medium or format, as long as you give appropriate credit to the original author(s) and the source, provide a link to the Creative Commons licence, and indicate if changes were made. The images or other third party material in this article are included in the article's Creative Commons licence, unless indicated otherwise in a credit line to the material. If material is not included in the article's Creative Commons licence and your intended use is not permitted by statutory regulation or exceeds the permitted use, you will need to obtain permission directly from the copyright holder. To view a copy of this licence, visit http://creativecommons.org/licenses/by/4.0/.

\section{References}

American Psychiatric Association. (1994). Diagnostic and statistical manual of mental disorders (4th ed.).

American Psychiatric Association. (2013). Diagnostic and Statistical Manual of Mental Disorders (Fifth ed.). American Psychiatric Publishing.

Amiet, C., Gourfinkel-An, I., Bouzamondo, A., Tordjman, S., Baulac, M., Lechat, P., Mottron, L., \& Cohen, D. (2008). Epilepsy in autism is associated with intellectual disability and gender: evidence from a meta-analysis. Biological Psychiatry, 64, 577-582. https://doi.org/ 10.1016/j.biopsych.2008.04.030.

Atun-Einy, O., \& Ben-Sasson, A. (2018). Pediatric allied healthcare professionals' knowledge and self-efficacy regarding ASD. Research in
Autism Spectrum Disorders, 47, 1-13. https://doi.org/10.1016/j. rasd.2017.12.001.

Austriaco, K., Aban, I., Willig, J., \& Kong, M. (2019). Contemporary trainee knowledge of autism: how prepared are our future providers? Frontiers in Pediatrics, 7, 165. https://doi.org/10.3389/fped.2019. 00165.

Bakare, M. O., Ebigbo, P. O., Agomoh, A. O., \& Menkiti, N. C. (2008). Knowledge about childhood autism among health workers (KCAHW) questionnaire: description, reliability and internal consistency. Clinical Practice and Epidemiology in Mental Health, 4, 17. https://doi.org/10.1186/1745-0179-4-17.

Bakare, M. O., Agomoh, A. O., Ebigbo, P. O., Eaton, J., Okonkwo, K. O., Onwukwe, J. U., \& Onyeama, G. M. (2009). Etiological explanation, treatability and preventability of childhood autism: a survey of Nigerian healthcare workers' opinion. Annals of General Psychiatry, 8, 6. https://doi.org/10.1186/1744-859X-8-6.

Bakare, M. O., Tunde-Ayinmode, M. F., Adewuya, A. O., Bello-Mojeed, M. A., Sale, S., James, B. O., Yunusa, M. A., Obindo, J. T., Igwe, M. N., Odinka, P. C., Okafor, C. J., Oshodi, Y. O., Okonoda, K. M., Munir, K. M., \& Orovwigho, A. O. (2015). Recognition of autism spectrum disorder (ASD) symptoms and knowledge about some other aspects of ASD among final year medical students in Nigeria, Sub-Saharan Africa. BMC Research Notes, 8, 454. https:// doi.org/10.1186/s13104-015-1433-0.

Baker, J. P. (2013). Autism at 70 - redrawing the boundaries. New England Journal of Medicine, 369, 1089-1091. https://doi.org/10. 1056/NEJMp1306380.

Beverly, B. L., \& Wooster, D. (2018). An interprofessional education initiative for allied health students preparing to serve individuals with autism spectrum disorders. Journal of Allied Health, 47(2), 90-95.

Boland, A., Cherry, G., \& Dickson, R. (2017). Doing a systematic review: a student's guide (2nd ed.).

Brown, A. B., \& Elder, J. H. (2014). Communication in autism spectrum disorder: a guide for pediatric nurses. Pediatric Nursing, 40(5), 219 225.

Chapman, A. L., Morgan, L. C., \& Gartlehner, G. (2010). Semiautomating the manual literature search for systematic reviews increases efficiency. Health Information and Libraries Journal, 27(1), 22-27. https://doi.org/10.1111/j.1471-1842.2009.00865.x.

Chiri, G., \& Warfield, M. E. (2012). Unmet need and problems accessing core health care services for children with autism spectrum disorder. Maternal and Child Health Journal, 16(5), 1081-1091. https://doi. org/10.1007/s10995-011-0833-6.

Clark, A., Browne, S., Boardman, L., Hewitt, L., \& Light, S. (2016). Implementing UK autism policy and national institute for health and care excellence guidance- assessing the impact of autism training for frontline staff in community learning disabilities teams. British Journal of Learning Disabilities, 44(2), 103-110. https:// doi.org/10.1111/bld.12116.

Cooper, K., Loades, M. E., \& Russell, A. J. (2018). Adapting psychological therapies for autism - therapist experience, skills and confidence. Research in Autism Spectrum Disorders, 45, 43-50. https:// doi.org/10.1016/j.rasd.2017.11.002.

Corsano, P., Cinotti, M., \& Guidotti, L. (2019). Paediatric nurses' knowledge and experience of autism spectrum disorders: An Italian survey. Journal of Child Health Care. https://doi.org/10.1177/ 1367493519875339.

Crane, L., Davidson, I., Prosser, R., \& Pellicano, E. (2019). Understanding psychiatrists' knowledge, attitudes and experiences in identifying and supporting their patients on the autism spectrum: Online survey. British Journal of Psychiatry Open, 5(3), 33. https:// doi.org/10.1192/bjo.2019.12.

Croen, L. A., Zerbo, O., Qian, Y., Massolo, M. L., Rich, S., Sidney, S., \& Kripke, C. (2015). The health status of adults on the autism 
spectrum. Autism, 19, 814-823. https://doi.org/10.1177/ 1362361315577517.

Dillenburger, K., McKerr, L., Jordan, J. A., Devine, P., \& Keenan, M. (2015). Creating an Inclusive Society... How close are we in relation to autism spectrum disorder? A general population survey. Journal of Applied Research in Intellectual Disabilities, 28(4), 330-340. https://doi.org/10.1111/jar.12144.

Ellias, S. D., \& Shah, H. R. (2019). A study of assessment of knowledge of childhood autism among medical students in Mumbai. Annals of Indian Academic Neurology, 22(2), 164-169. https://doi.org/10. 4103/aian.AIAN 486_17.

Elsabbagh, M., Divan, G., Koh, Y.-J., Kim, Y. S., Kauchali, S., Marcín, C., Montiel-Nava, C., Patel, V., Paula, C. S., Wang, C., Yasamy, M. T., \& Fombonne, E. (2012). Global prevalence of autism and other pervasive developmental disorders. Autism Research, 5(3), 160 179. https://doi.org/10.1002/aur.239.

Eseigbe, E. E., Nuhu, F. T., Sheikh, T. L., Eseigbe, P., Sanni, K. A., \& Olisah, V. O. (2015). Knowledge of childhood autism and challenges of management among medical doctors in Kaduna State, Northwest Nigeria. Autism Research and Treatment, 15, 1-6. https://doi.org/10.1155/2015/892301.

Gardner, M. R., Suplee, P. D., \& Jerome-D'Emilia, B. (2016). Survey of nursing faculty preparation for teaching about autism spectrum disorders. Nurse Educator, 41(4), 212-216. https://doi.org/10.1097/ NNE.0000000000000237.

Garg, P., Lillystone, D., Dossetor, D., Kefford, C., \& Chong, S. (2014). An exploratory survey for understanding perceptions, knowledge and educational needs of general practitioners (GPs) regarding autistic disorders in New South Wales (NSW Australia). Journal of Clinical and Diagnostic Research, 8(7). https://doi.org/10.7860/ JCDR/2014/8243.4527.

Gotham, K., Marvin, A. R., Taylor, J. L., Warren, Z., Anderson, C. M., Law, P. A., Law, J. K., \& Lipkin, P. H. (2015). Characterizing the daily life, needs, and priorities of adults with autism spectrum disorder from Interactive Autism Network data. Autism, 19(7), 794804. https://doi.org/10.1177/1362361315583818.

Greenhalgh, T., \& Peacock, R. (2005). Effectiveness and efficiency of search methods in systematic reviews of complex evidence: audit of primary sources. British Medical Journal, 331, 1064-1065. https:// doi.org/10.1136/bmj.38636.593461.68.

Greenland, S., \& O'Rourke, K. (2001). On the bias produced by quality scores in meta-analysis, and a hierarchical view of proposed solutions. Biostat, 2, 463-471. https://doi.org/10.1093/biostatistics/2.4. 463.

Hartley-McAndrew, M. D., Kathy Ralabate Doody, P., \& Jana Mertz, M. B. A. (2016). Knowledge of autism spectrum disorders in potential first-contact professionals. North American Journal of Medicine and Science, 7(3), 3 https://najms.com/index.php/najms/article/view/ 149.

Hayat, A. A., Meny, A. H., Salahuddin, N., Ahuja, K. R., \& Azeem, M. W. (2019). Assessment of knowledge about childhood autism spectrum disorder among healthcare workers in Makkah- Saudi Arabia. Pakistan Journal of Medical Science, 35(4), 951-957. https://doi. org/10.12669/pjms.35.4.605.

Hedley, D., Brewer, N., Nevill, R., Uljarević, M., Butter, E., \& Mulick, J. A. (2016). The relationship between clinicians' confidence and accuracy, and the influence of child characteristics, in the screening of autism spectrum disorder. Journal of Autism and Developmental Disorders, 46(7), 2340-2348. https://doi.org/10.1007/s10803-0162766-9.

Heidgerken, A. D., Geffken, G., Modi, A., \& Frakey, L. (2005). A survey of autism knowledge in a health care setting. Journal of Autism and Developmental Disorders, 35(3), 323-330. https://doi.org/10.1007/ s10803-005-3298-x.

Hofvander, B., Delorme, R., Chaste, P., Nydén, A., Wentz, E., Ståhlberg, O., Herbrecht, E., Stopin, A., Anckarsäter, H., Gillberg, C., Råstam,
M., \& Leboyer, M. (2009). Psychiatric and psychosocial problems in adults with normal-intelligence autism spectrum disorders. $B M C$ Psychiatry, 9(1), 35. https://doi.org/10.1186/1471-244X-9-35.

Hurlbutt, K., \& Chalmers, L. (2002). Adults with autism speak out: perceptions of their life experiences. Focus on Autism and Other Developmental Disabilities, 17(2), 103-111.

Iannuzzi, D., Rissmiller, P., Duty, S. M., Feeney, S., Sullivan, M., \& Curtin, C. (2019). Addressing a gap in healthcare access for transition-age youth with autism: a pilot educational intervention for family nurse practitioner students. Journal of Autism and Developmental Disorders, 49(4). https://doi.org/10.1007/s10803018-3846-9.

Igwe, M. N., Bakare, M. O., Agomoh, A. O., Onyeama, G. M., \& Okonkwo, K. O. (2010). Factors influencing knowledge about childhood autism among final year undergraduate medical, nursing and psychology students of University of Nigeria, Enugu State, Nigeria. Italian Journal of Pediatrics, 36(1), 44. https://doi.org/10. 1186/1824-7288-36-44.

Igwe, M. N., Ahanotu, A. C., Bakare, M. O., Achor, J. U., \& Igwe, C. (2011). Assessment of knowledge about childhood autism among paediatric and psychiatric nurses in Ebonyi state, Nigeria. Child and Adolescent Psychiatry and Mental Health, 5(1). https://doi.org/10. 1186/1753-2000-5-1.

Imran, N., Chaudry, M. R., Azeem, M. W., Bhatti, M. R., Choudhary, Z. I., \& Cheema, M. A. (2011). A survey of autism knowledge and attitudes among the healthcare professionals in Lahore, Pakistan. BMC Pediatrics, 11, 107. https://doi.org/10.1186/1471-2431-11107.

James, L. W., Pizur-Barnekow, K. A., \& Schefkind, S. (2014). Online survey examining practitioners' perceived preparedness in the early identification of autism. American Journal of Occupational Therapy, 68(1), 13-20. https://doi.org/10.5014/ajot.2014.009027.

Johnsson, G., Kerslake, R., Crook, S., \& Cribb, C. (2017). Investigation of training and support needs in rural and remote disability and mainstream service providers: Implications for an online training model. Australian Health Review, 41(6), 693-697. https://doi.org/ 10.1071/AH16132.

Jones, L., Goddard, L., Hill, E. L., Henry, L. A., \& Crane, L. (2014). Experiences of receiving a diagnosis of autism spectrum disorder: a survey of adults in the United Kingdom. Journal of Autism and Developmental Disorders, 44, 3033-3044. https://doi.org/10.1007/ s10803-014-2161-3.

Jones, K. B., Cottle, K., Bakian, A., Farley, M., Bilder, D., Coon, H., \& McMahon, W. M. (2016). A description of medical conditions in adults with autism spectrum disorder: a follow-up of the 1980s Utah/ UCLA Autism Epidemiologic Study. Autism, 20(5), 551-561. https://doi.org/10.1177/1362361315594798.

Joober, R., Schmitz, N., Annable, L., \& P, B. (2012). Publication bias: what are the challenges and can they be overcome? Journal of Psychiatry and Neuroscience, 37(3), 149-152. https://doi.org/10. 1503/jpn.120065.

Juni, P., Witschi, A., Bloch, R., \& Egger, M. (1999). The hazards of scoring the quality of clinical trials for meta-analysis. JAMA, 282, 1054-1060. https://doi.org/10.1001/jama.282.11.1054.

Kenny, L., Hattersley, C., Molins, B., Buckley, C., Povey, C., \& Pellicano, E. (2016). Which terms should be used to describe autism? Perspectives from the UK autism community. Autism, 20(4), 442-462. https://doi.org/10.1177/1362361315588200.

Khanna, R., Jariwala-Parikh, K., West-Strum, D., \& Mahabaleshwarkar, R. (2014). Health-related quality of life and its determinants among adults with autism. Research in Autism Spectrum Disorders, 8(3), 157-167. https://doi.org/10.1016/j.rasd.2013.11.003.

Kharti, G. K., Onta, S. R., Suresh, T., \& Choulagai, B. P. (2011). Knowledge and management practices of paediatricians about autism spectrum disorder in Kathmandu, Nepal. Journal of the 
Nepalese Paediatric Society, 31(2), 98-104. https://doi.org/10. 3126/jnps.v31i2.4640.

Kong, M. (2015). Diagnosis and history taking in children with autism spectrum disorder: dealing with the challenges. Frontiers in Pediatrics, 3, 55. https://doi.org/10.3389/fped.2015.00055.

Kuhlthau, K., Kovacs, E., Hall, T., Clemmons, T., Orlich, F., Delahaye, J., \& Sikora, D. (2013). Health-related quality of life for children with ASD: associations with behavioral characteristics. Research in Autism Spectrum Disorders, 7(9), 1035-1042. https://doi.org/10. 1016/j.rasd.2013.04.006.

Lai, M., \& Baron-Cohen, S. (2015). Identifying the lost generation of adults with autism spectrum conditions. The Lancet Psychiatry, 2(11), 1013-1027. https://doi.org/10.1016/S2215-0366(15)002771.

Lai, M. C., Lombardo, M. V., \& Baron-Cohen, S. (2014). Autism. The Lancet, 383(9920), 896-910. https://doi.org/10.1016/S01406736(13)61539-1.

Lai, M.-C., Kassee, C., Besney, R., Bonato, S., Hull, L., Mandy, W., Szatmari, P., \& Ameis, S. H. (2019). Prevalence of co-occurring mental health diagnoses in the autism population: a systematic review and meta-analysis. The Lancet Psychiatry, 6(10), 819-829. https://doi.org/10.1016/S2215-0366(19)30289-5.

Lever, A. G., \& Geurts, H. M. (2016). Psychiatric co-occurring symptoms and disorders in young, middle-aged, and older adults with autism spectrum disorder. Journal of Autism and Developmental Disorders, 46(6), 1916-1930. https://doi.org/10.1007/s10803-016-2722-8.

Lian, W. B., Ho, S. K. Y., Yeo, C. L., \& Ho, L. Y. (2003). General practitioners' knowledge on childhood developmental and behavioural disorders. Singapore Medical Journal, 44(8), 397-403.

Lugo-Marín, J., Magán-Maganto, M., Rivero-Santana, A., CuellarPompa, L., Alviani, M., Jenaro-Rio, C., Díez, E., \& Canal-Bedia, R. (2019). Prevalence of psychiatric disorders in adults with autism spectrum disorder: a systematic review and meta-analysis. Research in Autism Spectrum Disorders, 59, 22-33. https://doi.org/10.1016/j. rasd.2018.12.004.

Lüleci, N. E., Hidiroglu, S., Karavus, M., Celik, S., Cetiner, D., Koc, E., Kasikci, T. G., Sadirvan, Y. H., Karavus, A., \& Topuzoglu, A. (2016). A study exploring the autism awareness of first grade nursing and medical students in Istanbul, Turkey. The Journal of the Pakistan Medical Association, 66, 916.

Lyall, K., Croen, L., Daniels, J., Fallin, M. D., Ladd-Acosta, C., Lee, B. K., Park, B. Y., Snyder, N. W., Schendel, D., Volk, H., Windham, G. C., \& Newschaffer, C. (2017). The changing epidemiology of autism spectrum disorders. Annual Review of Public Health, 38, 81102. https://doi.org/10.1146/annurev-publhealth-031816-044318.

Mason, D., Ingham, B., Urbanowicz, A., Michael, C., Birtles, H., Woodbury-Smith, M., Brown, T., James, I., Scarlett, C., Nicolaidis, C., \& Parr, J. R. (2019). A systematic review of what barriers and facilitators prevent and enable physical healthcare services access for autistic adults. Journal of Autism and Developmental Disorders, 49(8), 3387-3400. https://doi.org/10. 1007/s10803-019-04049-2.

Matson, J. L., \& Kozlowski, A. M. (2011). The increasing prevalence of autism spectrum disorders. Research in Autism Spectrum Disorders, 5, 418-425. https://doi.org/10.1016/j.rasd.2010.06.004.

McGonigle, J. J., Migyanka, J. M., Glor-Scheib, S. J., Cramer, R., Fratangeli, J. J., Hegde, G. G., Shang, J., \& Venkat, A. (2014). Development and evaluation of educational materials for prehospital and emergency department personnel on the care of patients with autism spectrum disorder. Journal of Autism and Developmental Disorders, 44(5), 1252-1259. https://doi.org/10. 1007/s10803-013-1962-0.

Modesti, P. A., Reboldi, G., Cappuccio, F. P., Agyemang, C., Remuzzi, G., Rapi, S., Perruolo, E., \& Parati, G. (2016). Panethnic differences in blood pressure in Europe: a systematic review and meta-analysis. PLoS One, 11(1). https://doi.org/10.1371/journal.pone.0147601.
Mohammed, I. H., \& Majeed, B. N. (2019). Assessment of nurse's awareness about autism spectrum disorder in pediatric wards at Kirkuk public hospitals. Indian Journal of Public Health Research \& Development, 10(4), 579. https://doi.org/10.5958/0976-5506.2019. 00762.9.

Moher, D., Liberati, A., Tetzlaff, J., Altman, D. G., \& Group, T. P. R. I. S. M. A. (2009). Preferred Reporting Items for Systematic Reviews and Meta-Analyses: the PRISMA statement. PLoS Medicine, 6(7), 100097. https://doi.org/10.1371/journal.pmed.1000097.

Moher, D., Shamseer, L., Clarke, M., Ghersi, D., Liberati, A., Petticrew, M., Shekelle, P., Stewart, L. A., \& Group, P. R. I. M. S. A.-P. (2015). Preferred reporting items for systematic review and metaanalysis protocols (PRISMA-P) 2015 statement. Systematic Reviews, 4(1). https://doi.org/10.1186/2046-4053-4-1.

Muhammad, Z., Al-Deen, L. D., \& Muhsin, H. A. (2013). Knowledge about childhood autism among care providers in Baghdad. The Arab Journal of Psychiatry, 24(1), 27-31. https://doi.org/10.12816/ 0000095.

Murphy, D., \& McMorrow, K. (2015). View of autism spectrum conditions held by staff working within a high secure psychiatric hospital. The Journal of Forensic Practice, 17(3), 231-240. https://doi.org/ 10.1108/jfp-01-2015-0005.

Murphy, C. M., Wilson, C. E., Robertson, D. M., Ecker, C., Daly, E. M., Hammond, N., Galanopoulos, A., Dud, I., Murphy, D. G., \& McAlonan, G. M. (2016, July 7). Autism spectrum disorder in adults: Diagnosis, management, and health services development, Neuropsychiatric Disease and Treatment. Dove Press. https://doi. org/10.2147/NDT.S65455.

National Institute for Health and Care Excellence. (2012). https://www. nice.org.uk/guidance/cg142.

NICE. (2016). Overview $\mid$ Autism spectrum disorder in adults: Diagnosis and management | Guidance | NICE. NICE https://www.nice.org. uk/guidance/cg142.

NICE. (2017). Overview | Autism spectrum disorder in under 19s: Recognition, referral and diagnosis | Guidance | NICE. NICE $\mathrm{https://www.nice.org.uk/guidance/cg128.}$

Nicolaidis, C., Raymaker, D., McDonald, K., Dern, S., Boisclair, W. C., Ashkenazy, E., \& Baggs, A. (2012). Comparison of healthcare experiences in autistic and non-autistic adults: a cross-sectional online survey facilitated by an academic-community partnership. Journal of General Internal Medicine, 28(6), 761-769. https://doi.org/10. 1007/s11606-012-2262-7.

Nicolaidis, C., Raymaker, D., McDonald, K., Kapp, S., Weiner, M., Ashkenazy, E., Gerrity, M., Kripke, C., Platt, L., \& Baggs, A. (2016). The development and evaluation of an online healthcare toolkit for autistic adults and their primary care providers. Journal of General Internal Medicine, 31(10), 1180-1189. https://doi.org/ 10.1007/s11606-016-3763-6.

Portway, S. M., \& Johnson, B. (2005). Do you know I have Asperger's syndrome? Risks of a non-obvious disability. Health, Risk \& Society, 7(1), 73-83. https://doi.org/10.1080/09500830500042086.

Rahbar, M. H., Ibrahim, K., \& Assassi, P. (2011). Knowledge and attitude of general practitioners regarding autism in Karachi, Pakistan. Journal of Autism and Developmental Disorders, 41(4), 465-474. https://doi.org/10.1007/s10803-010-1068-x.

Rydzewska, E., Hughes-McCormack, L. A., Gillberg, C., Henderson, A., MacIntyre, C., Rintoul, J., \& Cooper, S. A. (2019). General health of adults with autism spectrum disorders - a whole country population cross-sectional study. Research in Autism Spectrum Disorders, 60, 59-66. https://doi.org/10.1016/j.rasd.2019.01.004.

Sampson, W. G., \& Sandra, A. E. (2018). Comparative study on knowledge about autism spectrum disorder among paediatric and psychiatric nurses in Public Hospitals in Kumasi, Ghana. Clinical Practice and Epidemiology in Mental Health, 14, 99-108. https://doi.org/10. 2174/1745017901814010099. 
Saqr, Y., Braun, E., Porter, K., Barnette, D., \& Hanks, C. (2017). Addressing medical needs of adolescents and adults with autism spectrum disorders in a primary care setting. Autism, 22(1), 51-61. https://doi.org/10.1177/1362361317709970.

Schaaf, R. C., Toth-Cohen, S., Johnson, S. L., Outten, G., \& Benevides, T. W. (2011). The everyday routines of families of children with autism: examining the impact of sensory processing difficulties on the family. Autism, 15, 373-389. https://doi.org/10.1177/ 1362361310386505.

Self, T., Coufal, K., \& Parham, D. (2010). Allied healthcare providers' role in screening for autism spectrum disorders. Journal of Allied Health, 39(3), 165-174.

Shaukat, F., Fatima, A., Zehra, N., Gulamhussein, M. A., \& Ismail, O. (2014). Assessment of knowledge about childhood autism among medical students from private and public universities in Karachi. The Journal of the Pakistan Medical Association, 64, 1331.

Stone, W. L. (1987). Cross-disciplinary perspectives on autism. Journal of Pediatric Psychology, 12(4), 615-630. https://doi.org/10.1093/ jpepsy/12.4.615.

Strunk, J. A. (2009). School nurses' knowledge of autism spectrum disorders. Journal of School Nursing, 25(6), 445-452. https://doi.org/ 10.1177/1059840509348221.

Systematic reviews: CRD's guidance for undertaking reviews in healthcare. (2009). Centre for Reviews and Dissemination.

Unigwe, S., Buckley, C., Crane, L., Kenny, L., Remington, A., \& Pellicano, E. (2017). GPs' confidence in caring for their patients on the autism spectrum: an online self-report study. British Journal of General Practice, 67(659), 445-452. https://doi.org/10. 3399/bjgp17X690449.

Westminster Commission on Autism. (2016). A spectrum of obstacles: an inquiry into access to healthcare for autistic people. https:// westminsterautismcommission.files.wordpress.com/2016/03/ ar1011_ncg-autism-report-july-2016.pdf

White, D., Hillier, A., Frye, A., \& Makrez, E. (2019). College students' knowledge and attitudes towards students on the autism spectrum.
Journal of Autism and Developmental Disorders, 49, 2699-2705. https://doi.org/10.1007/s10803-016-2818-1.

Will, D., Barnfather, J., \& Lesley, M. (2013). Self-perceived autism competency of primary care nurse practitioners. The Journal for Nurse Practitioners, 9(6), 350-355. https://doi.org/10.1016/j.nurpra.2013. 02.016 .

Williams, M. E., \& Haranin, E. C. (2016). Preparation of mental health clinicians to work with children with co-occurring autism spectrum disorders and mental health needs. Journal of Mental Health Research in Intellectual Disabilities, 9(1-2), 83-100. https://doi. org/10.1080/19315864.2016.1166302.

Wong, C., Odom, S. L., Hume, K. A., Cox, A. W., Fettig, A., Kucharczyk, S., Brock, M. E., Plavnick, J. B., Fleury, V. P., \& Schultz, T. R. (2015). Evidence-based practices for children, youth, and young adults with autism spectrum disorder: a comprehensive review. Journal of Autism and Developmental Disorders, 45(7), 1951-1966. https://doi.org/10.1007/s10803-014-2351-z.

Zerbo, O., Massolo, M. L., Qian, Y., \& Croen, L. A. (2015). A study of physician knowledge and experience with autism in adults in a large integrated healthcare system. Journal of Autism and Developmental Disorders, 45(12), 4002-4014. https://doi.org/10.1007/s10803$015-2579-2$.

Zwaigenbaum, L., Nicholas, D. B., Muskat, B., Kilmer, C., Newton, A. S., Craig, W. R., Ratnapalan, S., Cohen-Silver, J., Greenblatt, A., Roberts, W., \& Sharon, R. (2016). Perspectives of health care providers regarding emergency department care of children and youth with autism spectrum disorder. Journal of Autism and Developmental Disorders, 46(5), 1725-1736. https://doi.org/10. 1007/s10803-016-2703-y.

Publisher's Note Springer Nature remains neutral with regard to jurisdictional claims in published maps and institutional affiliations. 